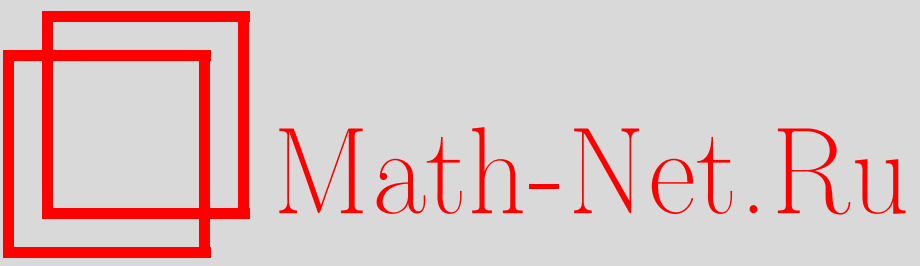

М.-М. Деза, М. И. Штогрин, Примитивные полициклы и гелицены, УМН, 1999, том 54, выпуск 6, 159-160

DOI: https://doi.org/10.4213/rm234

Использование Общероссийского математического портала Math-Net.Ru подразумевает, что вы прочитали и согласны с пользовательским соглашением

http://www.mathnet.ru/rus/agreement

Параметры загрузки:

IP : 54.172 .240 .79

26 апреля 2023 г., 15:32:33 


\title{
ПРИМИТИВНЫЕ ПОЛИЦИКЛЫ И ГЕЛИЦЕНЫ
}

\author{
М. ДЕЗА, М.И. Штогрин
}

Пусть $\left(r^{3}\right)$ - разбиение сферы $\mathbb{S}^{2}$ при $r=3,4,5$, евклидовой плоскости $\mathbb{R}^{2}$ при $r=6$ и плоскости Лобачевского $\mathbf{H}^{2}$ при $r \geqslant 7$ на правильные $r$-угольники с углами $\frac{2 \pi}{3}$ при вершинах.

ПРЕДЛОЖЕНИЕ. Край $r$-угольника является кратчайшим циклом в разбиении $\left(r^{3}\right)$.

Каждому разбиению $\left(r^{3}\right)$ однозначно сопоставим комбинаторно изоморфный ему абстрактньй двумерньй полиэдр $\mathbf{K}\left(r^{3}\right)$, составленный из правильных евклидовых $r$-угольников.

Плоский граф $G$ вместе со всеми его внутренними гранями (см. [2]) назовем примитивным nоли-r-циклом и обозначим его через $\Pi(G)$, если выполнены следующие три условия:

(1) гранища внешней грани $G$, совпадающая с границей $\Pi(G)$, есть простой цикл;

(2) все внутренние грани ограничены простыми циклами одной и той же длины $r$;

(3) пересечение любых двух внутренних граней либо есть одно ребро, либо пусто.

Если $\Pi(G)$ есть поли- $r$-цикл, то степень каждой вершины графа $G$ равна 2 или 3 , граф $G$ является 2 -связным, сам полищикл $\Pi(G)$ и все его (внутренние, [2]) грани изоморфны диску.

Назовем поли- $r$-цикл $\Pi(G)$ собственным, если граф $G$ является частичным подграфом реберного остова правильного разбиения $\left(r^{3}\right)$, и несобственным в противном случае. Несобственный поли- $r$-цикл $\Pi(G)$ назовем гелиценом над $\left(r^{3}\right)$, если существует комбинаторное отображение $\Pi(G) \rightarrow\left(r^{3}\right)$, при котором не менее двух ребер имеют один и тот же образ.

Теорема 1. Если планарный граф $G$ допускает на плоскости реализачию плоской карты (см. [2]) в виде полицикла П $(G)$, то все такие реализации изоморфны.

ЗАмечАниЕ. Реберный остов тетраэдра, куба, додекаэдра имеет соответственно 4, 6,12 изоморфных реализаций. У любого другого допустимого графа реализация единственна.

Существует единственный абстрактный двумерный полиэдр $\mathbf{P}(G)$, все грани которого суть евклидовы правильые $r$-угольники, комбинаторно изоморфный поли- $r$-циклу $\Pi(G)$.

ПримечаниЕ. Полиэдр $\mathbf{P}(G)$ состоит из таких же правильных $r$-угольников, как и полиэдр $\mathbf{K}\left(r^{3}\right)$. Поэтому отдельный $r$-угольник полиэдра $\mathbf{P}(G)$ допускает изометрическое отображение на $r$-угольник полиэдра $\mathbf{K}\left(r^{3}\right)$ (причем это отображение однозначно определяется указанием барицентрического треугольника и его образа при отображении). У полиэдров $\mathbf{P}(G)$ и $\mathbf{K}\left(r^{3}\right)$ изометричны пары смежных по внутренней стороне $r$-угольников и тройки сходящихся во внутренней вершине $r$-угольников. Как изометрия пары, так и изометрия тройки однозначно определяется по своему ограничению на одном $r$-угольнике.

ОСНовНАЯ ЛЕМма. Пусть П $(G)$ есть произвольный поли-r-цикл. Тогда существует непрерывное локально изометрическое клеточное отображсение $f: \mathbf{P}(G) \rightarrow \mathbf{K}\left(r^{3}\right)$.

Если $f$ является топологическим отображением полиэдра $\mathbf{P}(G)$ на его образ в $\mathbf{K}\left(r^{3}\right)$, то полицикл $\Pi(G)$ является собственным. Если же отображение $f$ полиэдра $\mathbf{P}(G)$ на его образ в $\mathbf{K}\left(r^{3}\right)$ является не топологическим отображением, а гомоморфизмом, то полицикл $\Pi(G)$ является гелиценом над $\left(r^{3}\right)$. Этим исчерпываются все возможности для отображения $f$.

ТЕОРема 2. Любой несобственный поли- - -цикл $\Pi(G)$ является гелиценом над $\left(r^{3}\right)$. Комбинаторное отображсние поличикла $\Pi(G)$ в разбиение $\left(r^{3}\right)$ однозначно определяется заданием образов фиксированной вериинь, инцидентного ей ребра и инцидентной ему грани.

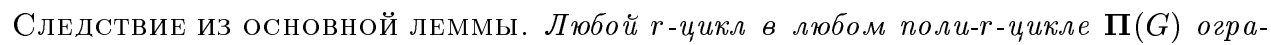
ничивает грань.

Работа выполнена при поддержке Российского фонда фундаментальных исследований (грант № 98-01-00251). 
В трех исключительных случаях $\Pi\left(3^{3}\right), \mathbf{\Pi}\left(4^{3}\right), \boldsymbol{\Pi}\left(5^{3}\right)$, когда $\mathbf{P}\left(3^{3}\right)=\mathbf{K}\left(3^{3}\right)-\mathbf{f}, \mathbf{P}\left(4^{3}\right)=$ $\mathbf{K}\left(4^{3}\right)-\mathbf{f}, \mathbf{P}\left(5^{3}\right)=\mathbf{K}\left(5^{3}\right)-\mathbf{f}$ соответственно, имеется один $r$-цикл, ограничивающий удаленную грань $\mathbf{f}$. Любой другой $r$-цикл в этих трех случаях, а также любой $r$-цикл в любом ином поли- $r$ цикле ограничивает грань самого полицикла $\Pi(G)$, т.е. внутреннюю грань плоского граффа $G$.

УтвеРЖДЕНИЕ. Если поли-г-цикл $\Pi(G)$ имеет не менее двух граней, то пересечение края хотя бы одной из граней с краем поли-r-цикла $\Pi(G)$ есть простая цепь.

В самом деле, край $\partial \Pi(G)$ поли- $r$-цикла $\Pi(G)$ в силу условия $(1)$ есть простой цикл $z$. Возьмем ребро $e_{1}$ из $z$. Оно принадлежит некоторой грани $\mathbf{f}_{1}$. Пусть $\partial \mathbf{f}_{1} \cap \partial \boldsymbol{\Pi}(G)$ не связно. Тогда будем перемешаться вдоль $z$ в определенном направлении, начиная с ребра $e_{1}$. Первое ребро $e_{2}$ из $z$, не принадлежащее $\mathbf{f}_{1}$, принадлежит грани $\mathbf{f}_{2}$. Если $\partial \mathbf{f}_{2} \cap \partial \Pi(G)$ не связно, идем вдоль $z$ далее. Первое ребро $e_{3}$ из $z$, не принадлежащее $\mathbf{f}_{2}$, принадлежит грани $\mathbf{f}_{3}$, отличной не только от $\mathbf{f}_{2}$, но и от $\mathbf{f}_{1}$, так как грань $\mathbf{f}_{2}$, примыкающая к краю полицикла $\boldsymbol{\Pi}(G)$ в разных местах, отгораживает $\mathbf{f}_{3}$ от $\mathbf{f}_{1}$. Если $\partial \mathbf{f}_{3} \cap \partial \mathbf{\Pi}(G)$ не связно, идем далее ... Через конечное число шагов получим грань $\mathbf{f}_{n}$, для которой пересечение $\partial \mathbf{f}_{n} \cap \partial \boldsymbol{\Pi}(G)$ связно. Так как $\Pi(G)$ содержит не менее двух граней, то $\partial \mathbf{f}_{n}$ не совпадает с $z$. Утверждение доказано.

ДОКАЗАТЕЛЬСТВО ОСНОВНОЙ ЛЕММЫ. Если полИэдр $\mathbf{P}(G)$ состоИт из одного $r$-угольника, а он равен $r$-угольнику из $\mathbf{K}\left(r^{3}\right)$, то лемма выполняется. Пусть лемма справедлива для всех полиэдров $\mathbf{P}(G)$ с числом граней, не превышаюшим $k-1$. Докажем ее для любого полиэдра $\mathbf{P}(G)$ с $k$ гранями, $k \geqslant 2$. В силу утверждения полиэдр $\mathbf{P}(G) \sim \boldsymbol{\Pi}(G)$ имеет такую грань $\mathbf{f}$, что пересечение $\partial \mathbf{f} \cap \partial \mathbf{P}(G)$ есть простая цепь $x$. Край $\partial \mathbf{f}$ грани $\mathbf{f}$ в силу условия (2) является простым циклом $x+y$. Простая цепь $y$ не пуста. Удалив из полиэдра $\mathbf{P}(G)$ грань $\mathbf{f}$, получим соответствующий графу $G-x$ полиэдр $\mathbf{P}(G-x)=\mathbf{P}(G)-\mathbf{f}$ с $k-1$ гранями. Край $\partial \mathbf{P}(G-x)$ полиэдра $\mathbf{P}(G)$ - $\mathbf{f}$ является простым циклом $z-x+y$. Проверено условие (1), а (2) и (3) верны по наследству. По предположению индукции имеется отображение $\mathbf{P}(G-x)$ в $\mathbf{K}\left(r^{3}\right)$. Возьмем цепь $y$ и ее образ при этом отображении. В силу условия (3) по всем ребрам цепи $y$ с гранью $\mathbf{f}$ смежны разные грани полиэдра $\mathbf{P}(G)$, принадлежащие полиэдру $\mathbf{P}(G-x)$. Все их образы в $\mathbf{K}\left(r^{3}\right)$ также различны и примыкают к образу цепи $y$, принадлежащему одному $r$-угольнику из $\mathbf{K}\left(r^{3}\right)$. Учитывая примечание, на этот $r$-угольник однозначно отображается грань $\mathbf{f}$, дополняющая полиэдр $\mathbf{P}(G-x)$ до полиэдра $\mathbf{P}(G)$. Лемма доказана.

ДоказАТЕЛЬСтво СЛЕДствия. В силу предложения и наличия отображения $f: \mathbf{P}(G) \rightarrow$ $\mathbf{K}\left(r^{3}\right)$ длина кратчайшего цикла в $\mathbf{P}(G)$ равна $r$. Рассмотрим любой $r$-цикл в $\mathbf{P}(G)$. Его образ при отображении $f$ есть $r$-цикл в $\mathbf{K}\left(r^{3}\right)$ и является краем плоского $r$-уголшника в $\mathbf{K}\left(r^{3}\right)$. Пусть этот плоский $r$-угольник не имеет прообраза при отображении $f$. Тогда все $r$ смежных с ним плоских $r$-угольников обязаны иметь прообразы, иначе образу $r$-цикла неоткуда было бы взяться. Им принадлежит $\varepsilon$-окрестность образа $r$-цикла, гомеоморфная колцу. Прообраз этого кольца при отображении $f$ есть опять кольцо, совпадающее с $\varepsilon$-окрестностью исходного $r$-цикла в $\mathbf{P}(G)$. Отсюда и в силу условия (1) следует, что исходный $r$-цикл исчерпьвает весь край полиэдра $\mathbf{P}(G)$. А так как полиэдр $\mathbf{P}(G)$ гомеоморфен диску, то он имеет вид $\mathbf{P}\left(3^{3}\right)=\mathbf{K}\left(3^{3}\right)-\mathbf{f}, \mathbf{P}\left(4^{3}\right)=\mathbf{K}\left(4^{3}\right)-\mathbf{f}$, $\mathbf{P}\left(5^{3}\right)=\mathbf{K}\left(5^{3}\right)-\mathbf{f}$, когда $r$-цикл является краем выкинутой из многогранника грани $\mathbf{f}$. Во всех остальных случаях $r$-цикл является краем наличной (внутренней, см. [2]) грани полиэдра $\mathbf{P}(G)$. Так как $\mathbf{P}(G) \sim \Pi(G)$, то следствие доказано.

Доказательство теоремы 1 вытекает из следствия из основной леммы.

\section{СПИСОК ЛИТЕРАТУРЫ}

[1] Deza M., Shtogrin M. I. // Voronoi's conference on analytic number theory and space tilings, Kyiv, September, 7-14, 1998. Abstracts. Kyiv, 1998. Р. 19-23. [2] Харари Ф. Теория графов. М.: Мир, 1973.

LIENS, Ecole Normale Supérieure and CNRS, Paris; Математический институт им. В. А. Стеклова РАН 